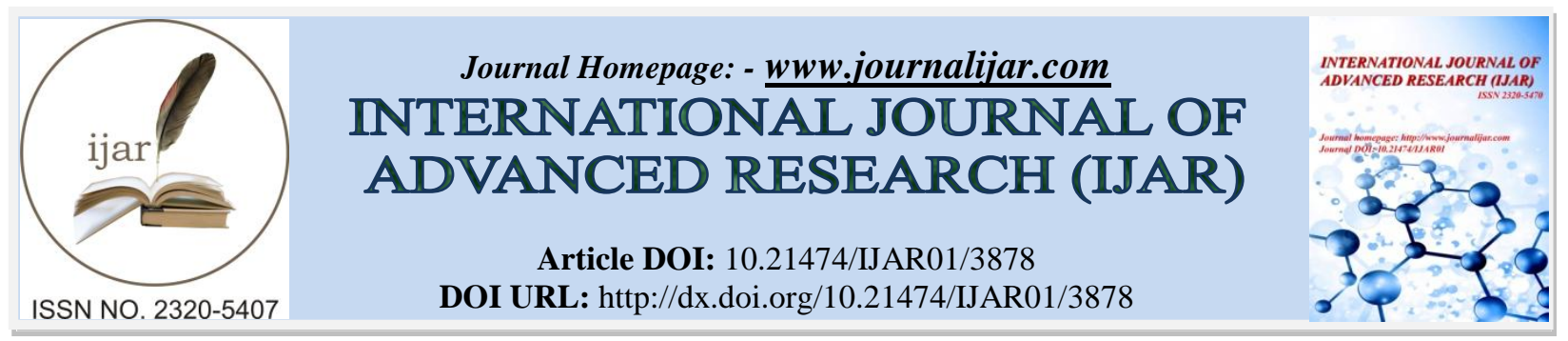

RESEARCH ARTICLE

\title{
REVIEW ON VARIOUS COMPLETE DENTURE TECHNIQUES.
}

\section{Nurul Syamimi binti Mohd Azlan Sunil ${ }^{1}$ and "Dr. Preetham Nittla².}

1. Bachelor of Dental Surgery (year 1), Saveetha Dental College, Chennai-600077.

2. Professor, Department of Prosthodontic, Saveetha Dental College and Hospitals, No.162, PH road, Chennai600077.

\section{Manuscript Info}

Manuscript History

Received: 04 February 2017

Final Accepted: 05 March 2017

Published: April 2017

\begin{abstract}
This review is performed to collect techniques in complete denture fabrication. Simpler methods of complete denture fabrication and technique helps to reduce appointments and offer better compliance to the patients with respect to time, logistic and expenses. The background of this review is the evolution of denture techniques that has led to reduced number of appointments without compromising the outcome and ensures patients' satisfaction with the treatment. The aim of this review is to compile the various techniques in complete denture fabrication.
\end{abstract}

Copy Right, IJAR, 2017,. All rights reserved.

\section{Introduction:-}

Over the past century, the technique of complete denture fabrication has evolved. Today, the most likely technique used in complete denture fabrication is the five appointment complete denture, which is the most suitable method of denture construction[1].

Complete denture teaching, about four decades ago is composed of lectures, followed by clinical work, and later on preclinical complete denture curriculum was established [2]. Preclinical work includes demonstrations for the purpose of effective performance by the students[3]. Now, living in the fast-paced world, advanced learning method has been implemented such as video lectures with demonstrations and visual aids have been introduced[4]. Provided with advanced learning method with technology in dental education, studies show that there are still significant ineptness in complete denture fabrication even after graduation. This study sparked the need to improve competency of complete denture fabrication in undergraduates. Using better clinical materials, improving training methods and by making the technique simpler, competency in fabricating complete dentures can be accomplished. Many authors have proposed simpler methods of complete denture fabrication[5].

Complete denture techniques has evolved over the past few decades that included nine visit denture, seven visit denture, five visit denture, BPS dentures, three appointment denture, two visit denture and single visit dentures.

\section{Nine Visit Denture:-}

Nine visit technique is the most time consuming complete denture fabrication method and was popular in 1950's [6].From this technique, complete denture fabrication started to evolve to simpler techniques. The nine visit denture technique consists of nine appointments, where the first two are diagnostic appointments and the remaining appointments are treatment appointments [7]. 
In the first diagnostic visit, a diagnostic impression is made with alginate using stock trays that is suitable for the patient. After the alginate impression is made, model plaster is pouredinthe impression to obtain diagnostic casts. In the second visit, the available inter-archis determined by doing tentative jaw relation. On the diagnostic cast, an acrylic denture base with occlusal rim is customized for the patient and articulated in the recorded vertical dimension and treatment planning is done.

Denture treatment starts from the third appointment. A primary impression is made to obtain a primary cast and to fabricate an acrylic custom tray. The custom tray is used for peripheral tracing and to carry the impression material for secondary impression.

In the fourth appointment, border moulding and secondary impressionsare made. The master cast is acquired by pouring a dental stone cast in the secondary impression. It is on this second cast the remains of the treatment is completed. Acrylic denture base and occlusal rims are constructed according to the standard dimensions.

Vertical jaw relation followed by facebow transfer is done during the fifth visit.

In the sixth visit, recording of centric relation is done. Gothic arch tracing records the centric relation and interocclusal records are obtained in centric and protrusive positions. By using the records, the articulator is programmed and condylar guidance is determined.

Anterior try in is done during the seventh appointment, where the maxillary and mandibular teeth are arranged. The incisal guidance is determined by the gliding of lower anterior teeth on the palatal aspect of maxillary teeth.

In the eight appointment, complete try in is done where the labial fullness, incisal exposure, smile line, vertical dimension, plane of occlusion and centric position are verified. After try in, the trial dentures are positioned on the master cast for final wax finishing and polishing.

In the ninth visit, the dentures are delivered where the aesthetics, phonetics, vertical dimension, centric relation are verified and occlusaldiscrepanciesare adjusted. The comfort of the patient wearing the denture is taken into account and the patients are instructed dental hygiene and oral hygiene measures.

This technique became unpopular due to the extensive treatment that consisted of many appointments. The requirement for reduced appointments prompted the advancement of improved and simplified techniques.

\section{Seven Visit Denture:-}

The seven visit denture was acknowledged as a strategy for education in dental schools and still followed in a few countries. It has lesser appointments and also known as balanced complete denture technique [8].

The first appointment is the primary impression visit from which a primary cast is obtained. The cast serves a role as diagnostic cast on which treatment arranging is done and custom tray is fabricated[7].

The second visit involves border moulding and final impression and is similar to the fourth appointment of the nine visit technique.

In the third appointment, the vertical jaw relation is recorded to determine the vertical dimension of the denture[7]. This is similar to the fifth appointment of the nine visit technique.

The next appointment is similar to appointment six of the nine visit techniques. Recording of horizontal jaw relation by gothic arch tracing is done in the fourth appointment

Similar to the seventh appointment of nine visit technique, the fifth appointment consist of verification of anterior teeth arrangement by aesthetics and phonetics.

Next, in the sixth visit, complete try in procedure is done and balanced contacts are verified in centric, protrusive and eccentric positions. This visit is comparable to the eighth appointment of nine visit denture. 
In the last visit, the denture is then inserted, similar to the ninth appointment of nine visit denture.

This techniques, like any other technique comes with its own advantages and disadvantages. Through balanced occlusal contacts, there is denture stability and also better chewing efficiency. The weakness of this technique is that different arrangements are required for this procedure. Besides that, the border moulding can be painful for the patient. Another downside of this technique is the horizontal jaw relation procedure was technique sensitive and time consuming.

\section{Five Visit Denture:-}

This technique was acquainted as an option with adjusted complete denture where tracing procedure was viewed as hard to perform. This has been adjusted in the undergraduate complete denture educational programs over the world $[9,10]$

In the first appointment, primary impression is made with impression compound or alginate. The lab procedure is like the seven visit denture.

The secondary impression is done with Zinc Oxide Eugenol impression paste or Polyvinylsiloxane impression material using a custom tray[11]. This step is done in the second appointment.

The third appointment is recording the vertical, horizontal jaw relation and inter occlusal records. This technique contrasts from the balanced occlusal method by keeping away from the tracing procedure and utilizing manual strategies for controlling the mandible to centric relation[7].

The fourth appointment is the try in appointment and only centric contacts can be verified during try in procedure. The last arrangement is when the patients gets the denture. Any occlusal discrepancies are balanced[7]. Additionally, oral cleanliness guidelines and dietary proposals are given. The patient is evaluated following a week. The benefits of this strategy are the decreased number of arrangements contrasted with the seven and nine visit technique, the commonality of the method and painful tracing procedure is kept away from. The drawback is the painful border moulding procedure is still followed in this technique.

\section{Four Appointment Denture BPS System:-}

This framework stresses the consolidation of different tooth forms to accomplish a congruous occlusion. The BPS denture has disentangled the impression procedures and jaw relation procedures to accomplish a balanced occlusion without trading off the stability of dentures[12].

The first appointment includes primary impression making and recording conditional jaw relation. Tray determination is done like different methods. Tentative jaw relation is recorded using centric trays. The cast are poured and tentative articulation is done in extraordinarily composed articulator. An extra feature of the articulator is its adaptability to incorporate Frankfurt's horizontal plane for jaw relation.

The second arrangement comprise of border moulding with polyvinylsiloxane material and recording the centric relation with Gnathometer. Any disparities in tentative vertical dimension measurements can be rectified at this stage. Inter occlusal records are acquired with CAD bite registration plate. Face bow exchange is done utilizing UTS face bow. The secondary casts are articulated according to jaw relation records obtained.

In the third arrangement, try in verification is finished. Centric relation is checked like the arrangement six of the seven visit denture. Adjusting contacts are checked.

The fourth and last arrangement is the insertion of the denture. The patient is reviewed following a week.

Like whatever other techniques, this technique accompanies its upsides and downsides. The benefits of this strategy are diminished clinical arrangements as the methods are streamlined, soundness of dentures by incorporating balanced occlusion in the teeth arrangement template, better biting productivity, advanced border moulding with polyvinylsiloxane material. Gnathometers fused into special trays and in this manner maintaining a strategic distance from uneasiness to the patient amid the tracing procedure and are anything but difficult to alter contrasted with the others. The hindrances are the expense of the denture, and the procedure is technique sensitive and needs 
extraordinary preparing. This technique is picking up prevalence as of late and there are no confirmations for disagreeability of this procedure.

\section{Three Visit Denture:-}

The three visit dentures are improved strategies for denture fabrication. There are three procedures that finishes denture fabrication in three arrangement. They are Tridenture, branching technique denture or Turbyfill denture are three appointment dentures.

The branching technique denture consist of three appointment denture. This technique can be considered as cost effective simplified technique.In the first appointment, an overextended alginate impression is made using prefabricated stock trays[13]. Anterior try in is done in the second appointment, and posterior teeth are not arranged and the posterior aspect of the denture remains like a block of acrylic[14]. Denture is inserted in the third appointment, where the soft liner is inserted during insertion. The patient uses this as a training denture for a period of three weeks after which teeth are incorporated in the posterior region[15].

Turbyfill is a modification of the branching technique where the posterior acrylic blocks are replaced with denture teeth. The procedure and the material used for this purpose are similar to the branching technique for the first appointment. Second appointment is the try in procedure where the posterior teeth are arranged like a conventional denture technique[14]. The third appointment includes denture insertion, where the soft liner is added during the insertion.

For fabricating highly accurate, stable and aesthetic dentures in three appointments, the effective system is the TriDenture. It is simple, accurate and results in fit and stability for the patient, and the reducing number of appointments.

\section{Two Visit Denture:-}

The two visit denture technique was developed to simplify the conventional complete denture fabrication.

In the first appointment, impression is made and inter alveolar distance is measured and sent to the laboratory to set the teeth arrangement and processing of the denture. Patient's teeth is selected based on bigger teeth or small row teeth.

The second appointment is when the denture is inserted. Patient satisfactory using this technique is comparable to the conventional denture claimed from the reported studies.

The advantages of this techniques include reduced cost and time. However, it is not a popular method as no clinical trials have been reported on this technique.

\section{Single Visit Dentures:-}

This technique is invented for immediate denture fabrication, which involves the Laurel's technique of complete denture fabrication that is done in about an hour.

The armamentarium includes prefabricated denture base with occlusal rims and teeth arranged on it. The impression making method is not known for this technique as it not clearly explained. Also, there is no strong evidence for claiming the success rate of this technique.

The advantage of this techniques are reduced cost, reduced chair side time and immediate denture fabrication. However, there is no reported proof of patient satisfactory with this technique. There is also no studies reported for success of this technique and is not popularly used.

\section{Conclusion:-}

Compilation of the various denture techniques in complete denture fabrication shows the evolution of denture techniques that led to reduced number of appointments, without compromising the outcome. Reduced appointments offer better compliance to the patients with respect to time, logistics and expenses. 


\section{References:-}

1. Clark, R., D. Radford, and M. Fenlon, The future of teaching of complete denture construction to undergraduates in the UK: is a replacement denture technique the answer? British dental journal, 2004. 196(9): p. 571-575.

2. Clark, R., The future of teaching of complete denture construction to undergraduates. British dental journal, 2002. 193(1): p. 13-14.

3. Faraone, K., P. Garrett, and E. Romberg, A blended learning approach to teaching pre-clinical complete denture prosthodontics. European Journal of Dental Education, 2013. 17(1): p. e22-e27.

4. Iwaki, M., et al., Live Broadcast Lectures on Complete Denture Prosthodontics at Tokyo Medical and Dental University: Comparison of Two Years. Journal of dental education, 2013. 77(3): p. 323-330.

5. Kawai, Y., et al., Do traditional techniques produce better conventional complete dentures than simplified techniques? Journal of dentistry, 2005. 33(8): p. 659-668.

6. Petropoulos, V.C. and B. Rashedi, Current concepts and techniques in complete denture final impression procedures. Journal of Prosthodontics, 2003. 12(4): p. 280-287.

7. Zarb, G.A., et al., Boucher's Prosthodontic Treatment for Edentulous Patients. 1997: Mosby.

8. Dubojska, A.M., G.E. White, and S. Pasiek, The importance of occlusal balance in the control of complete dentures. Quintessence international, 1998. 29(6).

9. Montero, J., R. Castillo-de Oyagüe, and A. Albaladejo, Curricula for the teaching of complete dentures in Spanish and Portuguese dental schools. Medicina oral, patologia oral y cirugia bucal, 2013. 18(1): p. e106.

10. Ozkurt, Z., I. Dikbas, and E. Kazazoglu, Predoctoral prosthodontic clinical curriculum for complete dentures: survey in Turkish dental schools. Journal of dental education, 2013. 77(1): p. 93-98.

11. Petropoulos, V.C. and B. Rashedi, Complete denture education in US dental schools. Journal of Prosthodontics, 2005. 14(3): p. 191-197.

12. Jiansheng, S., H. Jie, and Z. Yun, Effect of Occlusal Balance on Masticatory Efficiency of Complete Dentures. 2001: Stomatology.

13. Solanki, G., RECENT ADVANCEMENT IN COMPLETE DENTURE. International Journal of Biomedical Research, 2012. 3(1): p. 01-07.

14. Turbyfill, W., Dentures and partials or esthetic removable prosthetics. Current opinion in cosmetic dentistry, 1992: p. 75-79.

15. Murrell, G.A., The management of difficult lower dentures. The Journal of prosthetic dentistry, 1974. 32(3): p. 243-250. 